Revue de droit comparé du travail et de la sécurité sociale

1 | 2019

Doctrine

\title{
La surveillance des personnes assurées par les assureurs sociaux
}

\section{Anne-Sylvie Dupont}

\section{(2) OpenEdition}

1 Journals

Édition électronique

URL : https://journals.openedition.org/rdctss/1697

DOI : $10.4000 /$ rdctss. 1697

ISSN : 2262-9815

Éditeur

Centre de droit comparé du travail et de la sécurité sociale

Édition imprimée

Date de publication : 1 avril 2019

Pagination : 242-245

ISSN : 2117-4350

\section{Référence électronique}

Anne-Sylvie Dupont, «La surveillance des personnes assurées par les assureurs sociaux », Revue de droit comparé du travail et de la sécurité sociale [En ligne], 1 | 2019, mis en ligne le 01 novembre 2021, consulté le 13 novembre 2021. URL : http://journals.openedition.org/rdctss/1697 ; DOI : https:// doi.org/10.4000/rdctss. 1697

\section{(c) $(7)(9$}

Revue de droit comparé du travail et de la sécurité sociale est mise à disposition selon les termes de la Licence Creative Commons Attribution - Pas d'Utilisation Commerciale - Pas de Modification 4.0 International. 


\title{
ANNE-SYLVIE DUPONT
}

\author{
Universités de Neuchâtel et GenÈVE
}

\section{LA SURVEILLANCE DES PERSONNES ASSURÉES PAR LES ASSUREURS SOCIAUX}

L'année 2018 a été marquée, en Suisse, par l'adoption d'une disposition légale permettant aux assureurs sociaux nourrissant des soupçons à l'égard des personnes ayant demandé ou recevant des prestations sociales de faire observer ces dernières, soit par leurs propres collaborateurs, soit par des détectives privés.

Cette aventure législative a été initiée après que la Suisse ait été condamnée par la Cour européenne des droits de l'Homme dans une affaire concernant l'assuranceaccidents sociale, dans un arrêt rendu au mois d'octobre 2016. A l'origine de cette affaire, un assureur social avait en effet engagé un détective privé pour observer une assurée bénéficiant de prestations au long cours, dont le comportement lui paraissait suspect. Les griefs de l'assurée à ce propos avaient été rejetés jusqu'en dernière instance nationale. Les juges de Strasbourg ont pour leur part constaté que la législation suisse en matière d'assurances sociales, singulièrement en matière d'assurance-accidents, ne contenait pas de base légale satisfaisant aux réquisits de l'article 8 de la Convention européenne des droits de l'Homme (CEDH)1'. A la suite de l'arrêt strasbourgeois, les différents organes des assurances sociales ont annoncé suspendre les observations des personnes assurées. Dans le même temps, ils ont entrepris des démarches proactives auprès des milieux politiques afin qu'une solution législative soit trouvée le plus rapidement possible. La Caisse nationale d'assurance en cas d'accidents (CNA/SUVA), principal assureur-accidents du pays, s'est montrée particulièrement incisive à égard.

Le déroulement et - surtout - l'issue de cette affaire jettent une lumière inquiétante sur la vivacité des préoccupations sociales en Suisse (I), d'autant plus que le texte de la disposition adoptée laisse pour l'instant la porte ouverte à certaines dérives (II).

\section{I - L'ÉLABORATION DE LA DISPOSITION LÉGALE}

Le processus législatif helvétique n'est, traditionnellement, pas réputé pour sa rapidité, particulièrement dans le domaine de la sécurité sociale. En effet, la Suisse est un pays organisé en démocratie directe, doté, au niveau fédéral, d'un parlement bicaméral. L'histoire de l'élaboration de la législation en matière d'assurances sociales témoigne de la difficulté de trouver les consensus nécessaires à l'adoption de lois dans ce domaine ${ }^{2}$.

1 Affaire Vukota-Bojic c. Suisse du 18 octobre 2016, requête nº 61838.

2 Pour un aperçu rapide, cf. A.S. Dupont, «La Suisse et la sécurité sociale : ce qui prend du temps finit par être bien, in I. Daugareilh, M. Badel, La Sécurité sociale - Universalité et modernité - Approche de droit comparé, Paris 2019, p. 191 et p. 195. Pour une histoire plus détaillée de l'élaboration de la législation sociale en Suisse, cf. Ph. Gnaegi, Histoire, structure et financement des assurances sociales, $4^{\text {ème }}$ éd., Genève/Zurich/Bâle 2017, p. 37. 
S'agissant de la surveillance, les assureurs sociaux n'ont eu aucune difficulté pour trouver les relais politiques nécessaires pour qu'une disposition légale soit votée en quelques mois ${ }^{3}$. Les partis politiques traditionnels ayant renoncé à initier une récolte de signatures en vue d'obtenir un référendum populaire, c'est un groupement citoyen qui en a pris l'initiative, avec succès puisque les 50'000 signatures nécessaires ont été récoltées dans le délai de 100 jours prévu pour ce faire ${ }^{4}$. Après une campagne plutôt déséquilibrée en termes de fonds investis et hargneuse en termes de messages transmis, le peuple suisse s'est très nettement prononcé en faveur de la base légale proposée, à $64,7 \%$ des votants 5 , pour une participation de $47,5 \%$ de la population ayant le droit de vote 6

Outre l'influence des milieux économiques et des assureurs sociaux dans la campagne, il faut souligner que la campagne précédant la votation s'est caractérisée par plusieurs erreurs dans le matériel d'information distribué par le Gouvernement en vue de la votation, en particulier une erreur s'agissant du nombre de cas concernés annuellement ${ }^{7}$. Les erreurs ont ensuite été rectifiées, mais à une époque où une partie des votants s'était déjà exprimée, la Suisse connaissant le vote par correspondance.

\section{II - PROBLÈMES SOULEVÉS PAR L'APPLICATION DE LA DISPOSITION LÉGALE}

La disposition légale adoptée par le peuple ouvre un certain nombre de brèches dont il s'agira de voir comment elles seront utilisées - ou non - par les assureurs sociaux, et davantage encore par les tribunaux appelés à contrôler leurs décisions ${ }^{8}$.

En premier lieu, les circonstances permettant de mettre en œuvre une surveillance sont décrites de manière très large. Il faut que l'assureur social dispose « d'indices concrets laissant présumer qu'un assuré perçoit ou tente de percevoir indûment des prestations ». On notera, en premier lieu, que les indices ne doivent pas nécessairement porter sur un acte supposément frauduleux de la part de la personne assurée. II peut tout aussi bien s'agir, par exemple, d'une erreur de l'administration. A cet égard, une jurisprudence rendue récemment par le Tribunal fédéral, plus haute instance judiciaire en Suisse, offre un éclairage pour le moins préoccupant: il s'agissait d'une affaire dans laquelle il devait juger de la possibilité d'utiliser malgré tout comme moyen de preuve, dans une affaire donnée, un rapport de surveillance réalisé de manière illicite. Conformément à une jurisprudence

3 Pour plus de détails, cf. A.S. Dupont, «La surveillance des personnes assurées, ou l'urgence soudaine de légiférer », in Juristes démocrates de suisse (éd.), 40 ans des Juristes Démocrates de Suisse (JDS): résolu-e-s, impertinent-e-s, engagé-e-s., Berne 2018 (disponible sous https://www. djs-jds.ch/fr).

4 Cf. art. 141 al. 1 de la Constitution fédérale de la Confédération suisse du 18 avril 1999 (RS 101).

5 Cf. https://www.bk.admin.ch/ch/f/pore/va/20181125/det625.html.

6 Ils'agitd'un pourcentageassezélevé(cf.https://www.bfs.admin.ch/bfs/fr/home/statistiques/politique/ votations/participation.html).

7 Cf.Communiqué de presse du 7 novembre2018(https://www.admin.ch/gov/fr/accueil/documentation/ communiques.msg-id-72841.html). Cf. également Communiqué de presse du 21 novembre 2018 (https://www.admin.ch/gov/fr/accueil/documentation/communiques.msg-id-73044.html).

8 Le texte du futur art. 43a de la loi sur la partie générale du droit des assurances sociales (LPGA; RS 830.1), dont la date de l'entrée en vigueur n'a pas encore été communiquée, peut être consulté dans la Feuille fédérale (FF 2018 1469). 
établie, cela suppose avant toute chose que l'assureur social ait eu des soupçons sur le caractère justifié de la perception des prestations. En l'espèce, l'assureur-invalidité avait octroyé, en toute connaissance de cause, une rente d'invalide à une personne qui avait peut-être - un comportement contradictoire (exagération des plaintes), que les médecins n'avaient pas pu expliquer suffisamment, ni justifier d'un point de vue médical. L'assureur social avait ensuite, presque immédiatement après l'octroi de la rente, mis la personne assurée sous surveillance pour, sur la base du rapport d'observation, supprimer la rente après coup. Ne faisant aucune mention du caractère possiblement déloyal de l'attitude de l'assureur social, le Tribunal fédéral a simplement admis qu'en l'espèce, ce dernier était fondé à nourrir des soupçons ${ }^{9}$. Dans des affaires précédentes, le Tribunal fédéral avait déjà admis que le fait, pour une personne assurée, d'être active sur Facebook alors qu'elle alléguait des troubles psychiques, était de nature à induire des soupçons justifiant une surveillance ${ }^{10}$. Sur la base de ces jurisprudences, dont on doit s'attendre à ce que les tribunaux s'en inspirent pour l'application du nouveau droit, il est permis de penser que le recours à la surveillance sera validé de façon plutôt décomplexée.

Ensuite, il est intéressant de noter que le législateur a confié davantage de pouvoir à l'assureur social qu'aux autorités de la poursuite pénale. Contrairement à ce qui a pu être avancé dans le cadre de la campagne, et soutenu notamment par le Gouvernement, le fait est que la fraude à l'assurance sociale fait l'objet d'une norme pénale à l'article 148a du Code pénal ${ }^{11}$, qui réprime l'« obtention illicite de prestations », autrement dit des comportements intentionnels, au moins au stade du dol éventuel. Comme nous l'avons vu au paragraphe précédent, la surveillance en matière d'assurances sociales peut être ordonnée même en l'absence de tout comportement dolosif.

Conformément à la loi suisse, les autorités pénales qui entendent faire surveiller une personne soupçonnée de fraude à l'assurance sociale peuvent, dans le cadre de leurs prérogatives, " observer secrètement des personnes et des choses dans des lieux librement accessibles et effectuer des enregistrements audio et vidéo $»^{12}$. La surveillance dans des lieux privés, ou encore en utilisant d'autres moyens techniques, comme la géolocalisation, n'est possible que pour un certain nombre d'infractions, limitativement énumérées par le Code de procédure pénale, au nombre desquelles l'obtention illicite de prestations d'une assurance sociale (art. 148a CPP) ne figure pas ${ }^{13}$. II est vrai que les assureurs sociaux sont tenus, s'ils entendent recourir à la géolocalisation de la personne assurée, d'obtenir l'aval d'un juge, concession arrachée de haute lutte dans le cadre de la procédure législative ${ }^{14}$. Toujours est-il que sous cette réserve, l'accès à cette technologie leur est possible, alors que les autorités de la poursuite pénale ne pourraient en faire autant, même avec l'autorisation d'un juge, l'infraction pénale concernée ne figurant pas dans la liste des infractions pour lesquelles la loi pénale autorise le recours à des technologies particulières.

9 Cf. TF, arrêt 9C_264/2018 du 28 novembre 2018 (les arrêts non publiés du Tribunal fédéral sont disponibles sur le site Internet https://www.bger.ch/fr/index/juridiction.htm).

10 Cf. TF, arrêt 8C_616/2017 du 14 décembre 2017.

$11 \mathrm{CP}(\mathrm{RS} 311)$.

12 Cf. art. 282 al. 1 du Code de procédure pénale (CPP; RS 312.0).

13 Cf. art. 281 al. 4 cum 269 al. 2 CPP.

14 Cf. A.S. Dupont, (note 3), p. 76. 
Il est vrai aussi que les assureurs sociaux ne sont, pas davantage que les autorités de la poursuite pénale, habilités à observer les personnes assurées dans le domaine privé. La nuance réside ici dans le fait que la disposition légale adoptée en novembre dernier permet aux assureurs sociaux de faire surveiller les personnes assurées non seulement dans les lieux librement accessibles au public, mais aussi dans des lieux visibles depuis un lieu librement accessible. D’après les explications données par le Gouvernement dans le cadre de la votation, il s'agit, par exemple, d'un balcon ou d'un jardin visible depuis la rue, à l'exclusion d'une pièce fermée, par exemple l'intérieur d'un salon. Quoi qu'il en soit, un balcon serait considéré, dans le cadre d'une procédure pénale, comme un espace privé, de sorte qu'il faudrait obtenir une autorisation spéciale pour y effectuer des observations, autorisation qu'il ne serait pas possible d'obtenir en cas de soupçon de fraude à l'assurance, pour la même raison qu'au paragraphe précédent.

Finalement, la nouvelle disposition légale permettra aux assureurs sociaux, comme nous l'avons mentionné plus haut, de confier des missions d'observation à des détectives privés. Alors que cela n'était pas prévu dans le projet initial de la disposition, la loi apporte la précision - bienvenue - que ces derniers sont soumis au secret professionnel, et qu'ils ont l'interdiction d'utiliser à d'autres fins les informations récoltées dans le cadre de leur mandat. Dans l'ordonnance d'application, le Gouvernement a précisé que l'activité de surveillance pour le compte des assureurs sociaux était soumise à l'obtention préalable d'une autorisation. La délivrance de cette autorisation suppose, parmi d'autres conditions, que la personne qui la sollicite établisse qu'elle connaît les droits des assurés et les dispositions du droit pénal relatives à la surveillance, et qu'elle ait une connaissance suffisante du système de sécurité sociale et du droit des assurances sociales suisses. L'ordonnance ne précise pas comment cette preuve doit être apportée, en particulier quelles formations seront reconnues, ni par qui elles devront être dispensées. Le candidat à la surveillance pour le compte des assurances sociales doit en outre être au bénéfice d'une formation de policier ou d'« une formation équivalente ", sans que les critères permettant de juger de cette équivalence aient été précisés. En l'état, la formulation de l'ordonnance laisse la porte ouverte à une interprétation plutôt large, permettant de prendre en considération une formation militaire ou paramilitaire, y compris à l'étranger. Finalement, les motifs pour lesquels une personne au bénéfice d'une formation policière souhaiterait désormais se consacrer à la surveillance personnelle ne sont pas mentionnés comme faisant possiblement obstacle à la délivrance de l'autorisation. Ainsi, une révocation pour motifs disciplinaires, y compris graves, ne semble a priori pas dirimante.

II ressort du rapide panorama décrit ci-dessus que les bénéficiaires de prestations sociales en Suisse devront s'attendre, dès l'entrée en vigueur de la nouvelle réglementation, à pouvoir être surveillés en tout temps, y compris lorsqu'ils n'ont rien à se reprocher. Audelà des incertitudes créées par des dispositions légales et réglementaires formulées de manière vague et ouverte, l'adoption même de cette réglementation, l'âpreté du débat qui a précédé la votation et le résultat de cette dernière témoignent sans doute possible d'un rétrécissement de la vocation sociale de la Confédération helvétique. 